\title{
Enantioselective Cyanosilylation of Ketones Catalyzed by a Chiral Oxazaborolidinium Ion
}

\author{
Do Hyun Ryu and E. J. Corey* \\ Department of Chemistry and Chemical Biology \\ Harvard University, Cambridge, Massachusetts, 02138, USA \\ Department of Chemistry, Sungkyunkwan University, Suwon 440-746, Korea
}

$\underline{\text { Supplementary Materials }}$

Materials and Methods. Thin-layer chromatography (TLC) was performed using E. Merck silica gel $60 \mathrm{~F}_{254}$ precoated plates $(0.25 \mathrm{~mm})$. Flash chromatography was performed using Baker silica gel (40 $\mu \mathrm{m}$ particle size). NMR spectra were recorded on Varian Innova-500 or Mercury400 instruments and calibrated using residual undeuterated solvent as an internal reference. IR spectra were recorded on Avatar 360 FT-IR spectrometer. Low-resolution mass (CI) spectra were obtained by using a Platform II mass spectrometer. Analytical high performance liquid chromatography (HPLC) was performed on Agilent 1100 Series HPLC using the indicated chiral column. Gas chromatography (GC) analyses were performed on Hewlett-Packard 6850 Series GC System equipped with flame ionization detector using an Alltech Chiraldex $\gamma$-TA (30 m x $0.25 \mathrm{~mm}$ ) column. Toluene was distilled from sodium. Trimethylsilyl cyanide, acetophenone were distilled from calcium hydride. Cyclohexyl methyl ketone and pyruvic aldehyde dimethyl acetal were distilled from magnesium sulfate.

General Procedure for the Preparation and Use of Chiral Catalyst. A 100-mL, two-necked, round-bottomed flask equipped with a stir bar, a glass stopper and a 50-mL pressure-equalizing addition funnel (containing a cotton plug and ca. $10 \mathrm{~g}$ of $4 \mathrm{~A}$ molecular sieves, ${ }^{1}$ and functioning as a Soxhlet extractor) fitted on top with a reflux condenser and a nitrogen inlet adaptor was

\footnotetext{
${ }^{1}$ Molecular sieves (pellets) were dried in vacuo at $c a .200{ }^{\circ} \mathrm{C}$ with a gas burner for 10 min prior to use.
} 
charged with $(S)-(-)-\alpha, \alpha$-dimexyl-2-pyrrolidinemethanol ${ }^{2}(125.0 \mathrm{mg}, 0.404 \mathrm{mmol})$, tri- $o$ tolylboroxine $^{3}(47.5 \mathrm{mg}, 0.134 \mathrm{mmol})$ and $25 \mathrm{~mL}$ of toluene. The resulting solution was heated to reflux (bath temperature $\sim 145^{\circ} \mathrm{C}$ ). After $3 \mathrm{~h}$, the reaction mixture was cooled to $\mathrm{ca} .60{ }^{\circ} \mathrm{C}$ and the addition funnel and the condenser were quickly replaced with a short-path distillation head. The mixture was concentrated by distillation (air-cooling) to a volume of $c a$. $5 \mathrm{~mL}$. This distillation protocol was repeated three times by re-charging with $3 \times 5 \mathrm{~mL}$ of toluene. The solution was then allowed to cool to room temperature to give the oxazaborolidine precursor solution $(0.404 \mathrm{mmol})$ in toluene $(\mathrm{ca} .4 \mathrm{~mL})$. To the solution at $-25{ }^{\circ} \mathrm{C}$ was added trifluoromethanesulfonic acid $(29.8 \mu \mathrm{L}, 0.337 \mathrm{mmol})$ dropwise. After $10 \mathrm{~min}$ at $-25^{\circ} \mathrm{C}$, a colorless homogeneous catalyst solution was ready for use in the cyanosilylation reaction.

To methyldiphenylphosphine oxide $(79 \mathrm{mg}, 0.37 \mathrm{mmol})$ was added a catalyst solution in toluene $(4 \mathrm{~mL})$ at $0{ }^{\circ} \mathrm{C}$ and it was stirred for $5 \mathrm{~min}$ until methyldiphenylphosphine oxide dissolved in toluene completely. Trimethylsilyl cyanide $(0.67 \mathrm{~mL}, 5.0 \mathrm{mmol})$ was added to the catalyst solution in one portion and a methylketone $(3.37 \mathrm{mmol})$ solution in toluene $(1 \sim 3 \mathrm{~mL})$ was dropwise added to the solution. Then the reaction mixture was stirred under the reaction condition indicated in Table 1 and monitored by GC or TLC. When it was judged to be complete, volatile materials were evaporated in vacuo and water $(5 \mathrm{~mL})$ and pentane $(5 \mathrm{~mL})$ were added. The aqueous layer was extracted with pentane $(3 \times 5 \mathrm{~mL}){ }^{4}$ The combined extract was dried over anhydrous $\mathrm{Na}_{2} \mathrm{SO}_{4}$, filtered through glass filter and concentrated to afford the oily residue. The residue was passed through short silica gel column (gradient elution with 2-10\% EtOAc-hexanes) to afford the corresponding TMS protected cyanohydrin.

The corresponding racemic products for the determination of enantioselectivity were prepared by using ethylaluminum dichloride $(10 \mathrm{~mol} \%)$ as a Lewis acid catalyst.

\footnotetext{
${ }^{2}$ Mathre, D. J.; Jones, T. K.; Xavier, L. C.; Blacklock, T. J.; Reamer, R. A.; Mohan, J. J.; Jones, E. T. T.; Hoogsteen, K.; Baum, M. W.; Grabowski, E. J. J. J. Org. Chem.1991, 56, 751-762.

${ }^{3}$ Corey, E. J.; Shibata, T.; Lee, T. W. J. Am. Chem. Soc. 2002, 124, 3808-3809.

${ }^{4}$ After pentane extraction, the aqueous solution was treated with aqueous $2 \mathrm{~N} \mathrm{NaOH}$ solution and extracted with diethyl ether. The combined extract was dried over $\mathrm{Na}_{2} \mathrm{SO}_{4}$, concentrated and purified by column chromatography (elution with EtOAc) to afford pure ligand (95-99\% recovery).
} 


\section{Determination of Enantioselectivity and Absolute Configuration of the Cyanohydrin TMS Ethers Reported in Tables 1.}

Table 1, entry 1. Enantioselectivity of TMS ether was determined by GC analysis $\left(\gamma-\mathrm{TA}, 90{ }^{\circ} \mathrm{C}\right.$, $22 \mathrm{psi}$ ); retention times: 27.79 (major), $28.99 \mathrm{~min}$ (minor). The absolute configuration was determined by the sign of optical rotation: $[\alpha]_{\mathrm{D}}^{23}+13.3\left(c 2.0, \mathrm{CHCl}_{3}, 85 \%\right.$ ee $)$. [lit. $[\alpha]_{\mathrm{D}}^{25}+15.1$ (c $1.52, \mathrm{CHCl}_{3}$ ) for $R$-enantiomer in $90 \%$ ee ]; Hamashima, Y.; Kanai, M.; Shibasaki, M. J. Am. Chem. Soc. 2000, 122, 7412-7413.

Table 1, entry 2. Enantioselectivity of TMS ether was determined by GC analysis $\left(\gamma-\mathrm{TA}, 75^{\circ} \mathrm{C}\right.$, $22 \mathrm{psi}$ ); retention times: 25.24 (minor), $27.58 \mathrm{~min}$ (major). The absolute configuration was determined by the comparison of the sign of optical rotation: $[\alpha]_{\mathrm{D}}^{23}+27.6\left(c 2.0, \mathrm{CHCl}_{3}, 96 \%\right.$ ee $)$. [lit. $[\alpha]_{\mathrm{D}}-16.8\left(c \quad 2.16, \quad \mathrm{CHCl}_{3}\right)$ for $R$-enantiomer of 2-trimethylsilyloxy-2(dipropyloxymethyl)propanenitrile in $92 \%$ ee ]; Tian, S.-K.; Hong, R.; Deng, L. J. Am. Chem. Soc. 2003, 125, 9900-9901.

Table 1, entry 3. Enantioselectivity of TMS ether was determined by GC analysis $\left(\gamma-\mathrm{TA}, 90{ }^{\circ} \mathrm{C}\right.$, $22 \mathrm{psi}$ ); retention times: 21.70 (major), $23.10 \mathrm{~min}$ (minor). The absolute configuration was determined by the sign of optical rotation: $[\alpha]_{\mathrm{D}}^{23}+21.5\left(c 1.0, \mathrm{CHCl}_{3}, 83 \%\right.$ ee $) \cdot\left[\right.$ lit. $[\alpha]_{\mathrm{D}}^{25}+21.9$ (c 1.18, $\mathrm{CHCl}_{3}$ ) for $R$-enantiomer in $93 \%$ ee ]; Hamashima, Y.; Kanai, M.; Shibasaki, M. J. Am. Chem. Soc. 2000, 122, 7412-7413.

Table 1, entry 4. Enantioselectivity of TMS ether was determined by GC analysis ( $\gamma$-TA, 120 ${ }^{\circ} \mathrm{C}, 22 \mathrm{psi}$ ); retention times: 25.19 (major), $26.79 \mathrm{~min}$ (minor). The absolute configuration was determined by the sign of optical rotation: $[\alpha]_{\mathrm{D}}^{23}+15.3\left(c 0.33, \mathrm{CHCl}_{3}, 81 \%\right.$ ee $)$. $\left[\right.$ lit. $[\alpha]_{\mathrm{D}}+29.5$ (c $1.04, \mathrm{CHCl}_{3}$ ) for $R$-enantiomer of 2-trimethylsilyloxy-2-(4'-chlorophenyl)propanenitrile in 92\% ee ]; Hamashima, Y.; Kanai, M.; Shibasaki, M. J. Am. Chem. Soc. 2000, 122, 7412-7413. 
Table 1, entry 5. Enantioselectivity of TMS ether was determined by GC analysis ( $\gamma$-TA, 140 ${ }^{\circ} \mathrm{C}, 22 \mathrm{psi}$ ); retention times: 42.80 (major), $46.98 \mathrm{~min}$ (minor). The absolute configuration was determined by the sign of optical rotation: $[\alpha]_{\mathrm{D}}^{23}+20.5\left(c 1.0, \mathrm{CHCl}_{3}, 96 \%\right.$ ee $)$. [lit. $[\alpha]^{20}{ }_{\mathrm{D}}+16.2$ ( $c$ 1.67, $\mathrm{CHCl}_{3}$ ) for $R$-enantiomer in $88 \%$ ee ]; Deng, H.; Isler, M. P.; Snapper, M. L.; Hoveyda, A. H. Angew. Chem. Int. Ed. 2002, 41, 1009-1012.

Table 1, entry 6. Enantioselectivity of TMS ether was determined by GC analysis ( $\gamma$-TA, 130 ${ }^{\circ} \mathrm{C}, 22 \mathrm{psi}$ ); retention times: 19.26 (major), $21.10 \mathrm{~min}$ (minor). The absolute configuration was determined by analogy with $(+)-(R)-2-$ Trimethylsilyloxy-2-(4'-nitrophenyl)propanenitrile : $[\alpha]_{\mathrm{D}}^{23}$ +13.1 ( $c 2.0, \mathrm{CHCl}_{3}, 95 \%$ ee $)$.

Table 1, entry 7. Enantioselectivity of TMS ether was determined by GC analysis $(\gamma$-TA, 130 ${ }^{\circ} \mathrm{C}, 22 \mathrm{psi}$ ); retention times: 16.69 (minor), 16.96 min (major). The absolute configuration was determined by the sign of optical rotation: $[\alpha]_{\mathrm{D}}^{23}-7.0\left(c 2.0, \mathrm{CHCl}_{3}, 32 \%\right.$ ee $)$. [lit. $[\alpha]^{21}{ }_{\mathrm{D}}+22.6(c$ 1.09, $\mathrm{CHCl}_{3}$ ) for $R$-enantiomer in $91 \%$ ee ]; Deng, H.; Isler, M. P.; Snapper, M. L.; Hoveyda, A. H. Angew. Chem. Int. Ed. 2002, 41, 1009-1012.

\section{Physical Data of TMS Ethers of Cyanohydrin:}

(R)-2-Trimethylsilyloxy-2-cyclohexylpropanenitrile (Table 1, entry 1). The physical and spectral data were identical to those previously reported for this compound ${ }^{5}:{ }^{1} \mathrm{H}$ NMR $(500 \mathrm{MHz}$, $\left.\mathrm{CDCl}_{3}\right) \delta 1.95-1.98(\mathrm{~m}, 1 \mathrm{H}), 1.81-1.83(\mathrm{~m}, 3 \mathrm{H}), 1.67-1.69(\mathrm{~m}, 1 \mathrm{H}), 1.52(\mathrm{~s}, 3 \mathrm{H}) 1.48(\mathrm{tt}, 1 \mathrm{H}, J=$ 12.0, 3.0 Hz), 1.04-1.28 (m, 5H), $0.23(\mathrm{~s}, 9 \mathrm{H}) ;[\alpha]_{\mathrm{D}}^{23}+13.3\left(\right.$ c 2.0, $\mathrm{CHCl}_{3}, 85 \%$ ee) .

(S)-2-Trimethylsilyloxy-2-(dimethoxymethyl)propanenitrile (Table 1, entry 2). ${ }^{1} \mathrm{H}$ NMR $\left(400 \mathrm{MHz}, \mathrm{CDCl}_{3}\right) \delta 4.13(\mathrm{~s}, 1 \mathrm{H}), 3.54(\mathrm{~s}, 3 \mathrm{H}), 3.51$ (s, 3H), $1.52(\mathrm{~s}, 3 \mathrm{H}), 0.23(\mathrm{~s}, 9 \mathrm{H}) ;{ }^{13} \mathrm{C} \mathrm{NMR}$

\footnotetext{
${ }^{5}$ Hamashima, Y.; Kanai, M.; Shibasaki, M. J. Am. Chem. Soc. 2000, 122, 7412-7413.
} 
$\left(100 \mathrm{MHz}, \mathrm{CDCl}_{3}\right) \delta$ 120.4, 107.6, 72.4, 57.8, 57.7, 23.2, 1.2(x3); FTIR (neat) 1254, 1164, 1088, 1028; LRMS (CI) calcd for $\left[\mathrm{C}_{9} \mathrm{H}_{23} \mathrm{~N}_{2} \mathrm{O}_{3} \mathrm{Si}\right]\left(\left[\mathrm{MNH}_{4}\right]^{+}\right)$: 235; found 235 ; $[\alpha]_{\mathrm{D}}^{23}+27.6(c$ 2.0, $\mathrm{CHCl}_{3}, 96 \%$ ee).

(R)-2-Trimethylsilyloxy-2-phenylpropanenitrile (Table 1, entry 3). The physical and spectral data were identical to those previously reported for this compound ${ }^{5}:{ }^{1} \mathrm{H} \mathrm{NMR}(500 \mathrm{MHz}$, $\left.\mathrm{CDCl}_{3}\right) \delta$ 7.53-7.56 (m, 2H), 7.38-7.42 (m, 2H), 7.33-7.37 (m, 1H), $1.86(\mathrm{~s}, 3 \mathrm{H}), 0.18(\mathrm{~s}, 9 \mathrm{H})$; $[\alpha]_{\mathrm{D}}^{23}+21.5\left(c 1.0, \mathrm{CHCl}_{3}, 83 \%\right.$ ee $)$.

(R)-2-Trimethylsilyloxy-2-(4'-bromophenyl)propanenitrile (Table 1, entry 4). ${ }^{1} \mathrm{H}$ NMR (400

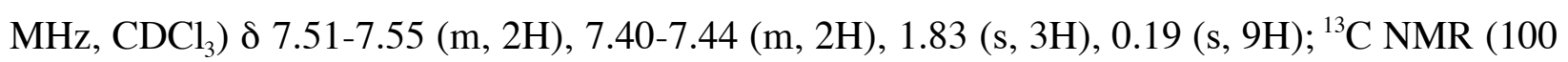
$\left.\mathrm{MHz}, \mathrm{CDCl}_{3}\right) \delta 141.2,131.7(\mathrm{x} 2), 126.3(\mathrm{x} 2), 122.7,121.1,71.0,33.4,1.0(\mathrm{x} 3)$; FTIR (neat) 1266, 1011, 735; LRMS (CI) calcd for $\left[\mathrm{C}_{12} \mathrm{H}_{20} \mathrm{BrN}_{2} \mathrm{OSi}\right]\left(\left[\mathrm{MNH}_{4}\right]^{+}\right): 315,317$; found 315,$317 ;[\alpha]_{\mathrm{D}}^{23}$ +15.3 ( $c 0.33, \mathrm{CHCl}_{3}, 81 \%$ ee).

(R)-2-Trimethylsilyloxy-2-(4'-nitrophenyl)propanenitrile (Table 1, entry 5). The physical and spectral data were identical to those previously reported for this compound ${ }^{6}:{ }^{1} \mathrm{H}$ NMR (400 $\left.\mathrm{MHz}, \mathrm{CDCl}_{3}\right) \delta$ 8.24-8.28 (m, 2H), 7.71-7.75 (m, 2H), 1.87 (s, 3H), $0.23(\mathrm{~s}, 9 \mathrm{H}) ;{ }^{13} \mathrm{C}$ NMR (100 $\left.\mathrm{MHz}, \mathrm{CDCl}_{3}\right) \delta 148.9,148.0,125.7(\mathrm{x} 2), 123.9(\mathrm{x} 2), 120.6,70.8,33.4,1.0(\mathrm{x} 3) ;[\alpha]_{\mathrm{D}}^{23}+20.5(c$ $1.0, \mathrm{CHCl}_{3}, 96 \%$ ee).

(R)-4-(1-Cyano-1-trimethylsilyloxyethyl)phenyl trifluoromethanesulfonate (Table 1, entry 6). ${ }^{1} \mathrm{H}$ NMR (400 MHz, $\mathrm{CDCl}_{3}$ ) $\delta$ 7.63-7.66 (m, 2H), 7.30-7.34 (m, 2H), 1.86 (s, 3H), 0.22 (s, 9H); ${ }^{13} \mathrm{C}$ NMR (100 MHz, $\mathrm{CDCl}_{3}$ ) $\delta 149.4,142.7,126.7(x 2), 121.6(x 2), 120.9,118.7$ (q, $J=319$ $\mathrm{Hz}), 70.8,33.4,1.0(\mathrm{x} 3)$; FTIR (neat) 1426, 1210, 1138, 841; LRMS (CI) calcd for $\left[\mathrm{C}_{13} \mathrm{H}_{16} \mathrm{~F}_{3} \mathrm{NO}_{4} \mathrm{Si}\right]\left([\mathrm{M}]^{+}\right): 367$; found $367 ;[\alpha]_{\mathrm{D}}^{23}+13.1\left(c 1.0, \mathrm{CHCl}_{3}, 95 \%\right.$ ee $)$.

(R)-2-Trimethylsilyloxy-2-(4'-methoxyphenyl)propanenitrile (Table 1, entry 7). The physical and spectral data were identical to those previously reported for this compound ${ }^{6}:{ }^{1} \mathrm{H}$

\footnotetext{
${ }^{6}$ Deng, H.; Isler, M. P.; Snapper, M. L.; Hoveyda, A. H. Angew. Chem. Int. Ed. 2002, 41, 1009-1012.
} 
NMR (500 MHz, $\left.\mathrm{CDCl}_{3}\right) \delta$ 7.44-7.48 (m, 2H), 6.89-6.92 (m, 2H), 3.83 (s, 3H), 1.85 (s, 3H), 0.16 $(\mathrm{s}, 9 \mathrm{H}) ;[\alpha]_{\mathrm{D}}^{23}-7.0\left(\right.$ c 2.0, $\mathrm{CHCl}_{3}, 32 \%$ ee) .

\section{Synthesis of $(R)$-4-Nitroatrolactic Acid.}

(R)-2-Hydroxy-2-(4'-nitrophenyl)propanamide. $(R)$-2-Trimethylsilyloxy-2-(4'-nitrophenyl) propanenitrile (310 mg, $1.17 \mathrm{mmol}, 96 \%$ ee) was suspended in concentrated $\mathrm{HCl}(37 \%, 3 \mathrm{~mL})$. For $30 \mathrm{~min}, \mathrm{HCl}$ gas was bubbled through the reaction mixture until it became a clear solution. Then it was diluted with water $(20 \mathrm{~mL})$ and the aqueous phase was extracted with ethyl acetate (4 X $3 \mathrm{~mL}$ ). The combined extract was dried over sodium sulfate and concentrated in vacuo to give pale yellow oily residue. The residue was purified by column chromatography (gradient elution with 30-100\% EtOAc-hexanes) to afford $225 \mathrm{mg}(91 \%)$ of product as a white solid. Recrystallization from a mixture solvent (EtOAc and dichloromethane) provided pure $(R)-2-$ hydroxy-2-(4'-nitrophenyl)propanamide: $\mathrm{mp} 132-133{ }^{\circ} \mathrm{C}$; ${ }^{1} \mathrm{H}$ NMR $\left(400 \mathrm{MHz}\right.$, acetone- $\left.d_{6}\right) \delta$ 8.18-8.22 (m, 2H), 7.91-7.95 (m, 2H), 7.27 (brs, 1H), 6.64 (brs, 1H), 1.79 (s, 3H); ${ }^{13} \mathrm{C}$ NMR (100 MHz, acetone- $\left.d_{6}\right) \delta 176.7,153.2,148.0,127.5(x 2), 123.6(x 2), 76.7,27.8$; FTIR (neat) 3467, 3355, 1667, 1515, 1345; LRMS (CI) calcd for $\left[\mathrm{C}_{9} \mathrm{H}_{10} \mathrm{~N}_{2} \mathrm{O}_{4}\right]\left([\mathrm{MH}]^{+}\right)$: 211 ; found $211 ;[\alpha]_{D}^{23}+2.6$ ( $c$ 1.0, acetone, $>99 \%$ ee). Enantioselectivity was determined by HPLC analysis using a Chiralcel OD (Daicel) column (5\% $i$-PrOH in hexanes for elution; $1.0 \mathrm{~mL} / \mathrm{min} ; \lambda 254 \mathrm{~nm}$ ); retention times: $50.2 \mathrm{~min}$ (major), $54.7 \mathrm{~min}$ (minor).

(R)-4-Nitroatrolactic Acid. A solution of amide (113 mg, $0.54 \mathrm{mmol})$ in $12 \mathrm{~N} \mathrm{HCl} \mathrm{(2} \mathrm{mL)} \mathrm{was}$ stirred at $80^{\circ} \mathrm{C}$ for $2 \mathrm{~h}$. After cooling, the solution was concentrated in vacuo and the residue was treated with water $(5 \mathrm{~mL})$ and ethyl acetate $(2 \mathrm{~mL})$. The aqueous phase was extracted with ethyl acetate $(3 \times 2 \mathrm{~mL})$. The combined extract was dried over sodium sulfate and concentrated in vacuo to give $112 \mathrm{mg}$ of product (99\%) as a white solid. Recrystallization from a mixture solvent (EtOAc and dichloromethane) provided pure $(R)-(-)$-4-nitroatrolactic acid: $\mathrm{mp} 90-91{ }^{\circ} \mathrm{C} ;{ }^{1} \mathrm{H}$ NMR (400 MHz, acetone- $\left.d_{6}\right) \delta$ 8.22-8.24 (m, 2H), 7.91-7.94 (m, 2H), $1.81(\mathrm{~s}, 3 \mathrm{H}) ;{ }^{13} \mathrm{C}$ NMR 
$\left(100 \mathrm{MHz}\right.$, acetone- $\left.d_{6}\right) \delta 175.5,151.8,148.1,127.6(x 2), 123.9(x 2), 76.1,27.7$; FTIR (neat) $3446,3114,1717,1519,1347$; LRMS (CI) calcd for $\left[\mathrm{C}_{9} \mathrm{H}_{9} \mathrm{NO}_{5}\right]\left([\mathrm{M}]^{+}\right): 211$; found $211 ; \quad[\alpha]_{D}^{23}$ -37.8 ( $c$ 1.0, acetone, $>99 \%$ ee). The absolute configuration was determined by analogy with commercial $(R)-(-)$-atrolactic acid (Lancaster, $98+\%):[\alpha]^{20}{ }_{\mathrm{D}}-49(c$ 2.0, water). 